\title{
Role of lipid droplets in diatoms for biofuel production
}

\author{
Rishanpreet Kaur, Lovepreet Kaur, Shristy Gautam* \\ Department of Molecular Biology and Genetic Engineering, School of Bioengineering and Biosciences, Lovely Professional University, Punjab, India.
}

\section{ARTICLE INFO \\ Article history: \\ Received on: March 18, 2021 \\ Accepted on: June 15, 2021 \\ Available online: September 01,2021}

Key words:

Diatom, fatty acids, lipid droplets, transesterification, triacylglycerol

\begin{abstract}
Diatoms have gained popularity due to their practical applications in pharmaceuticals, biotechnology, biofuel production, cosmetic products, and valuable food constituents. Diatoms have high lipid content; they can produce approximately $60 \%$ of their cellular mass as triacylglycerol (TAG) under certain growth conditions. Stress conditions, such as nutrient limitation, trigger accumulation of reserved compounds (i.e., lipids and chrysolaminarin) in diatoms. A complete understanding of TAG metabolism is required for biofuel production. They accumulate lipids as lipid droplets in cytoplasm. Lipid droplets have distinct intercellular compartments in which diatoms accumulate neutral lipids. Lipid droplets have proteins on their surface, and these proteins could be important for TAG metabolism, but a comprehensive study of lipid body-associated proteins is still pending. Lipid accumulation in diatoms can be enhanced using phytohormones, other hormone-derived compounds, and molecular engineering. Diatoms are rich in fatty acids, especially polyunsaturated fatty acids (PUFAs), which are beneficial for animal and plant growth. PUFAs extraction from diatoms has more advantages than from fish due to the fast growth of diatoms and feasible application of biotechnology on them to manipulate their genetic material. The present review focuses on the role of lipids, its accumulation, and extraction from diatoms by various methods.
\end{abstract}

\section{INTRODUCTION}

Diatoms are unicellular eukaryotic organisms characterized by a surrounding silica cell wall and yellow brown pigmentation [1]. Silicon cell walls are assumed to play a role in the growth and productivity of diatoms [2]. The origin of diatoms might be older than its first definitive evidence in the early Jurassic period. The earliest known freshwater diatoms appeared in the second epoch of the Paleogene period. By the first geological epoch of the Neogene Period, both marine and freshwater floras were diverse and numerous taxa were formed fundamentally, the same as the current living species [1]. Diatoms acquire chloroplast by secondary endosymbiosis; hence, the recent diatoms have related chloroplast to chloroplast of red algae. The chloroplast of diatoms contains chlorophyll a, c1, and c2 [3]. Plastids of diatoms have four membranes: two membranes are of the organelle itself and the outer two represent the plastid endoplasmic reticulum. Diatoms

*Corresponding Author

Shristy Gautam, Department of Molecular biology and Genetic

Engineering, School of Bioengineering and Biosciences, Lovely

Professional University, Phagwara, India.E-mail: shristy.g@gmail.com live in both fresh and seawater plus they could be found in aerosols and soil. Traditionally, diatoms are divided into two subgroups: pennate diatoms, which are bilaterally symmetric (pennales), and centric diatoms, which are radially symmetric (centrales) [4].

These photosynthetic autotrophs can be symbiotic with photosynthetic organisms [3]. Up to $20 \%$ of carbon dioxide is fixed by diatoms, making them the primary producers in fresh and seawater [5]. Diatoms use solar energy to build up organic molecules through carbon dioxide fixation. Carbon dioxide fixation is carried out by the Calvin-Bensson-Bassham cycle, in which the catalytic enzyme rubisco coverts carbon dioxide into ribulose-1,5-bisphosphate [6]. Diatoms need carbon dioxide for growth and metabolism. Additionally, carbon is required for storage compound synthesis. This suggests that an increase in carbon availability may improve lipid accumulation. Based on this hypothesis, studies have been conducted to test the relativity, and the outcome of some of these studies was positive. They have abundant amounts of carotenoids, isoprenoids, sterols, and lipids, and some are detected with toxins and other metabolites [4].

A lipid droplet is an organelle consisting of a neutral lipid [e.g., triacylglycerol (TAG)] core surrounded by a polar lipid 
monolayer. This organelle is conserved among all eukaryotes [7]. Diatoms synthesize lipids to maintain their buoyancy as lipids are more lightweight than other chemical constituents of these organisms and enable them to live suspended in the photic zone [4]. According to lipid droplet biogenesis, accumulation of neutral lipid to form accretion disk within endoplasmic reticulum takes place prior to its budding off into the cytoplasm [7]. Lipid droplets are associated with other organelles such as mitochondria, nucleus, endosomes, endoplasmic reticulum, peroxisome, vacuoles, and plastids via direct contact, proximity, and vesicular transport. There is a possibility that these inert lipid droplets are involved in active metabolic processes which are coordinated with other cellular compartments and respond to variations in cellular and environmental conditions [7].

According to the study conducted on Phaeodactylum tricornutum, under nitrate-starved conditions, the carbon fluxes decrease sharply into all major sinks and a major decrease was observed into proteins and least was into lipids. The largest factor preventing the industrial biodiesel production at prices competitive with fossil fuels is balance between growth rate and lipid content [8]. Diatoms are imperative for marine nutrition because they produce large amounts of polyunsaturated fatty acids (PUFAs), for example, eicosapentaenoic acid (EPA). Health and reproductive achievements of many marine organisms are affected by the amount and composition of lipids in phytoplankton. Abiotic factors such as temperature and low phosphorous level can affect lipid composition in diatoms. However, the complex ecosystem dynamics hinder the identification the degree to which specific abiotic factors influence the change in lipid composition in aquatic organisms. A study has been conducted on P. tricornutum and Chlamydomonas muelleri to examine the effect of temperature on their fatty acid composition. The fatty acids 14:0, 16:0, 16:1, 16:2, 16:3, 18:0, 18:1(n-9)c, 18:2(n-6), and 20:5(n-3) were detected in $P$. tricornutum; in addition to these fatty acids, $18: 1(n-9)$ $\mathrm{t}$ and 20:4(n-6) were detected in C. muelleri. An increase in temperature above optimal growth temperature for 2 hours had a higher effect on fatty acid composition and degree of saturation in C. muelleri than in P. tricornutum. However, according to protein assay analysis, not $C$. muelleri but $P$. tricornutum was undergoing stress while lipids were affected in both [9].

Diatoms have shown their significance in several applications. Because of their industrial scale culture conditions, growth, quantity, and socioeconomic considerations, diatoms are considered as a suitable raw material for bioactive metabolites production. Molecular genetic tools and different chemical approaches have been facilitating the identification of these components [3]. This review paper provides an overview on lipids, fatty acids and TAG, lipid accumulation, lipid proteome, and lipid extraction.

\subsection{Lipids, Fatty Acid, and TAG}

Diatoms are a substantial source of bioactive compounds. In addition to bioactive compounds, diatoms can generate a large variety of lipids. On an average, diatoms can achieve lipid content to $25 \%$ of dry weight. However, lipid content can increase under unfavorable environmental conditions such as high temperature nutrient availability or nutrient starvation. Diatoms hold a high value in biotechnology industry for lipid production [10]. Lipids are the primary storage material in diatoms [11]. Generally, fatty acids vary from $\mathrm{C} 14: 0$ to $\mathrm{C} 22: 6$ in diatoms. Docosahexaenoic acid (DHA), EPA, palmitic acid (C16:0), palmitoleic acid (C16:1 n-7), and myristic acid (14:0) are most common fatty acids. Fatty acid chains usually have two to three double bonds and are rarely more than six. Fatty acid profile is similar among many known algal species, but fatty acid content varies among species, it is mostly dependent on strain and culture conditions [10].

PUFAs are bioactive substances, straight chain fatty acid of approximately 18-22 carbon, and contain two or more double bonds. These are mainly classified into two principal families $n-3$ (or $\omega-3$ ) and $n-6$ (or $\omega-6$ ) that are derived biosynthetically from $\alpha$-linolenic acids (ALA, 18:3n-3) and linoleic acids (18:2n-2), respectively. $n-3$ PUFAs are essential nutrients and these are beneficial for health and growth in animals. Stearidonic acid (18:4n-3), DHA, 22:6n-3, ALA, 18:3n-3, and EPA, 20:5n-3 represent $n-3$ PUFAs; arachidonic acid (20:4n-6) and $\gamma$-linoleic acid (18:3n-6) represent $n-6$ PUFAs. DHA and EPA are good for vision, psychiatric disorder treatment, and cardiovascular system, while C18n-3 PUFAs do not confer such health benefits. Studies have revealed the correlation between larger ratios of $n-6$ to $n-3$ PUFAs with increased pathogenesis of many diseases, including coronary heart disease.

In aquaculture, DHA and EPA are essential nutrients for larvae of many shrimp, fish, and bivalve organisms [12]. PUFA content is imperative indices for aquatic feed nutritional value evaluation due to their potential to maintain a high reproductive rate and larval growth rate in marine and freshwater organisms. PUFAs have several benefits in humans and animals, so there is significant need of PUFA supplementation. However, PUFA resources are limited, and this restricts their wide application. Due to no substantial examples of land plants accumulating EPA and DHA, marine fish are generally reported as main source of PUFA. Different circumstances can affect the quality of fish oil, such as species, season, stemming, etc. Approximately $20 \%$ $30 \%$ EPA and DHA can be obtained from fish oil, but there are many unresolved issues. Fish accumulate PUFAs by PUFA-rich microalgae ingestion that means fish are not real producers of PUFA. Marine microalgae are the emerging PUFA resource; these have application in biofuel production. Extraction of PUFA from diatoms exhibits more perks than from fish. Firstly, the advantage of diatoms over fish is their high PUFA content. Secondly, these are unicellular microalgae with fast growth. Third, biotechnological means can be feasibly applied on diatoms to manipulate their metabolism to promote the synthesis of PUFAs. Abiotic factors such as light and temperature play a crucial role in cell growth and metabolite synthesis. The optimum growth temperatures for some microalgae have been determined to range from $16^{\circ} \mathrm{C}$ to $27^{\circ} \mathrm{C}$, such as $20^{\circ} \mathrm{C}$ is the optimum temperature for $P$. tricornutum. EPA, 20:5n-3 is the major component of fatty acid produced by P. tricornutum [3].

Aquatic species program targeted the identification of most promising, naturally occurring feedstock for biodiesel production. In this program, diatoms were shown to produce up to $60 \%$ of their cellular mass as TAG under certain growth conditions [13]. TAGs 
are main storage lipids; these are rich in energy and represent one of the major lipid classes of highly productive and fast-growing diatoms. Photosynthesis in light forms of TAG are then stored in systolic bodies and reused during polar lipid biosynthesis in the dark [4]. Transesterification reaction was used to convert TAGs into biodiesel [14]. TAG production was tried to be improved using genetic engineering, but it was hindered by lack of appropriate molecular biology tools to carry out the manipulations and genetic information. The optimal TAG production occurs in environmental stress conditions such as low temperature or nutrient deficiency. Due to lack of information on systematic comparisons of the yield and molecular makeup of TAG produced under these different stresses in the same species, it means there is a possibility that different environmental stresses have different effects on cellular components needed for TAG synthesis (Fig. 1) [13].

\subsection{Lipid Accumulation}

The main storage of lipids is lipid droplets in cytoplasm and are stored in chloroplast in less quantity [5]. The substantial applications of microalgae lipids in pharmaceuticals, biofuels production, and cosmetic products are the main reason to attract popularity. Lipid accumulation in microalgae has its productivity superior to crop plants as these accumulate lipid up to $73 \%$ of their dry weight. The photosynthetic efficiency and high lipid accumulation in diatoms have been proven as a good source of lipid in comparison to other microalgae. According to various studies, diatoms have the efficiency to accumulate lipid in nutrient limiting conditions. Phaeodactylum tricornutum, C. muelleri, and Thalassiosira weissflogii have shown their ability to increase the lipid accumulation after nitrogen starvation. Under sodium chloride and silicon limiting conditions, Chaetoceros gracilis has the ability to accumulate lipids up to $73 \%$. Modulation in lipid synthesis signaling pathway, phytohormone, and other hormonederived compounds can be useful in enhancing lipid accumulation in diatoms.

Molecular engineering can enhance lipid accumulation in diatoms using genetic transformation. For example, $P$. tricornutum has

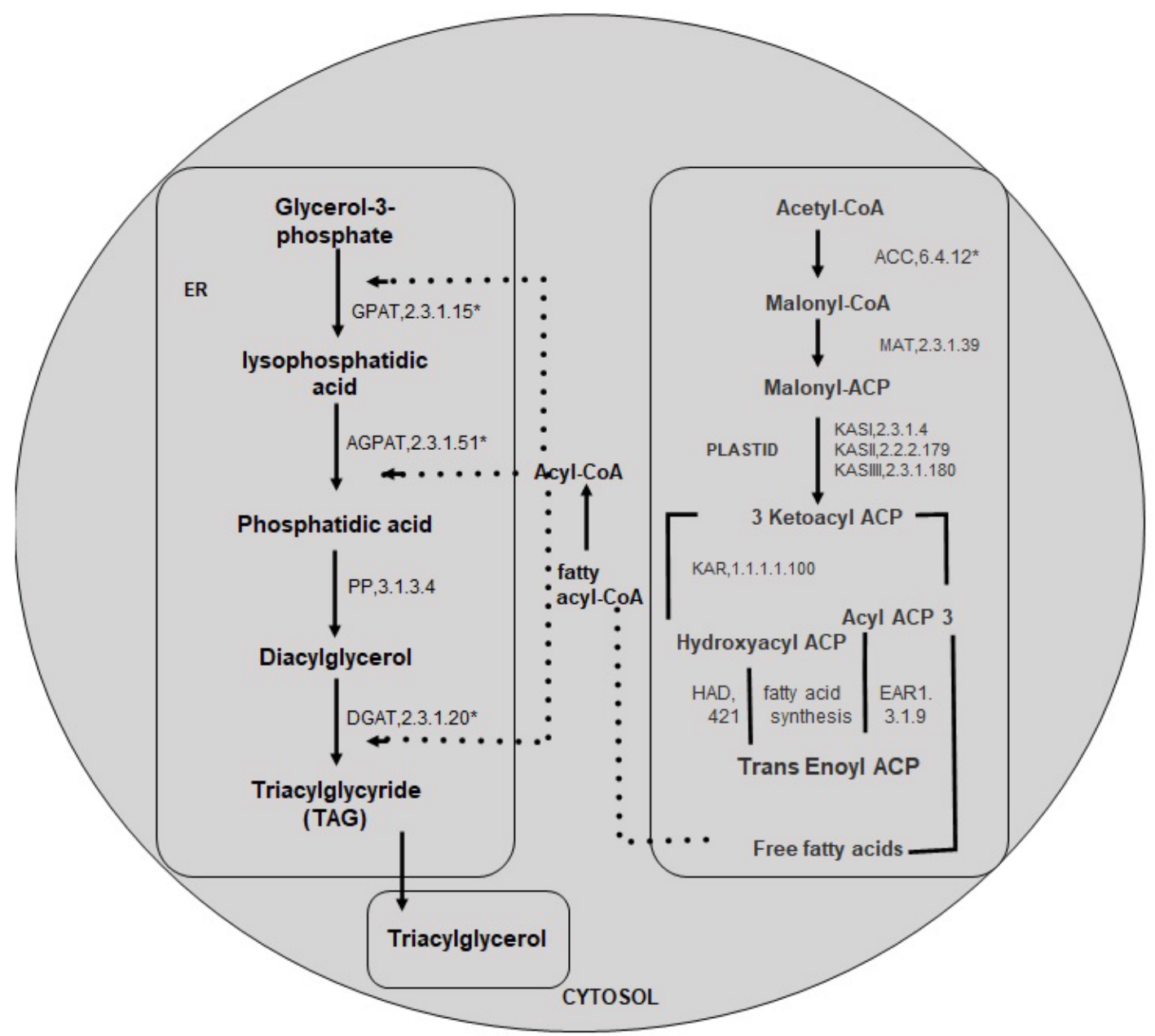

Figure 1. Schematic representation of TAG synthesis in diatoms. ER =, endoplasmic reticulum; G3PDH =, glycerol-3phosphate dehydrogenase; $\mathrm{ACC}=$, acetyl-coA carboxylase; GPAT =, glycerol-3-phosphate acyltransferase; MAT =, malonylcoA-AcP transferase; AgPAT =, 1-acylglycerol-3-phosphate acyltransferate; KAS =, 3-KETOACYL-AcP synthase; PP =, phosphate acyltransferase; KAr =, 3-Keyoacyl-AcP reductase; DgAT =; diacylglycerol acyltransferase; hAD =, 3-hydroxyacylAcP; Ear =, enoyl-AcP reductase (modified from [32]) 
shown increased lipid accumulation by overexpressing several genes involved in TAG biosynthesis. Environmental stresses can induce lipid biosynthesis [4]. It was observed that PUFAs and neutral lipids production is simulated by nutrient deficiencies such as phosphorous, silicon, and nitrogen; environment stresses such as salinity, physical stimuli, temperature, light intensity, etc. Moreover, there was an increase in the expression of genes involved in lipid biosynthesis under nitrogen and silicon unavailability [15]. Nitrogen (N)-deprived cells are more suitable for biofuel production due to the high proportion of saturated fatty acids in them [16]. Genetic engineering can help to enhance lipid production, such as in the study of $P$. tricornutum, the gene encoding the plastidic thioesterase was overexpressed which enhanced the lipid content from 78 to $133 \mathrm{mg} / \mathrm{g} /$ dry weight [2].

\subsection{Lipid Proteome}

Lipid droplet proteins have demonstrated to carry out various functions such as regulation of intracellular trafficking, lipid droplet formation, stabilization, degradation, and interaction between lipid droplets and other organelles [17]. Proteomics of lipid droplets is not completely explored yet due to many obstacles such as proteome of lipid droplets differs in cell type, strain, growth conditions, etc. and due to different location of lipid droplets within the cell. Lipid droplet proteins studies have been conducted on Naviculales [18], Fistulifera solaris [19], and four studies have been conducted on P. tricornutum [20-23]. Proteins coordinate several functions in most of the organelle. In plants and animals, proteins play an imperative role in determining the size and stability of lipid bodies.

Major lipid droplet proteins (MLDP) and lipid droplet surface proteins are present in microalgae [19]. MLDP regulates lipid droplet size because decreases in MLDP cause an increase in lipid droplet size. Fluorophore tagging, such as gene florescent protein, is used to demonstrate lipid body localization [24]. The lipid droplets in P. tricornutum were localized with a heterologous expressed Green fluorescent protein (GFP) tagged lipid droplets (LD) (GFP-tagged LD) protein from Haematococcous pluvialis. However, the low-resolution efficiency of optical microscope is an obstacle as it has difficulty in differentiating physically associated proteins or the proteins located just near to it on an adjacent

\section{A}

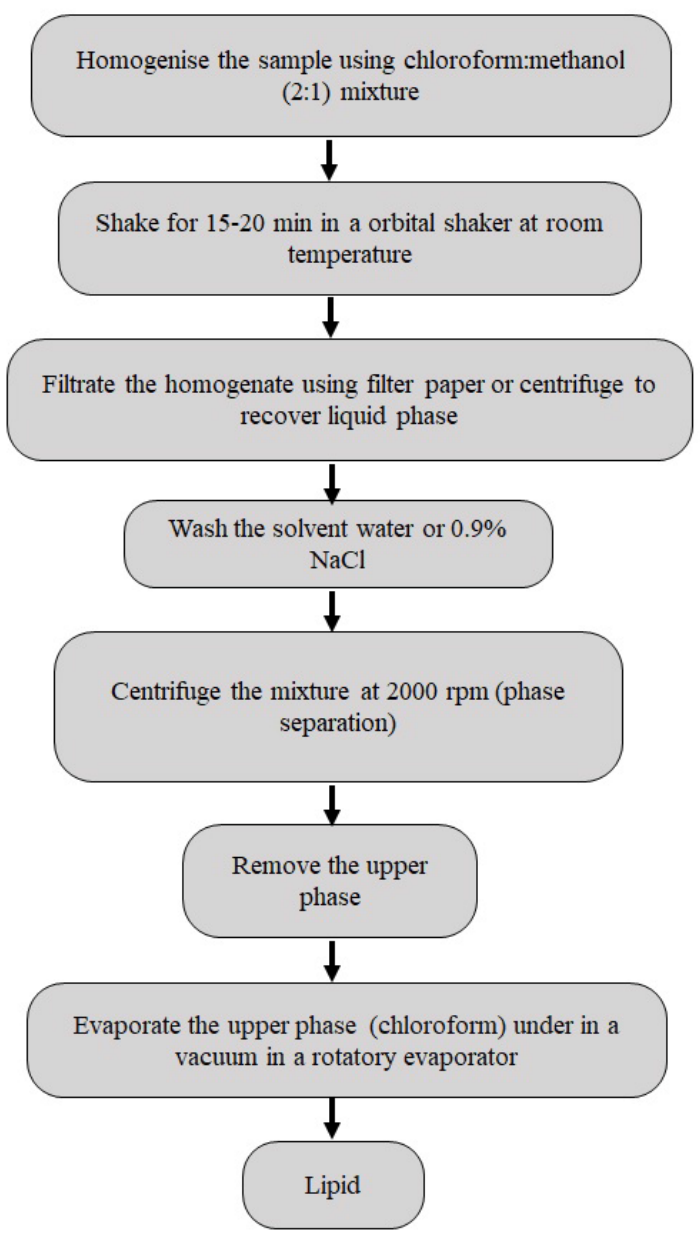

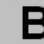

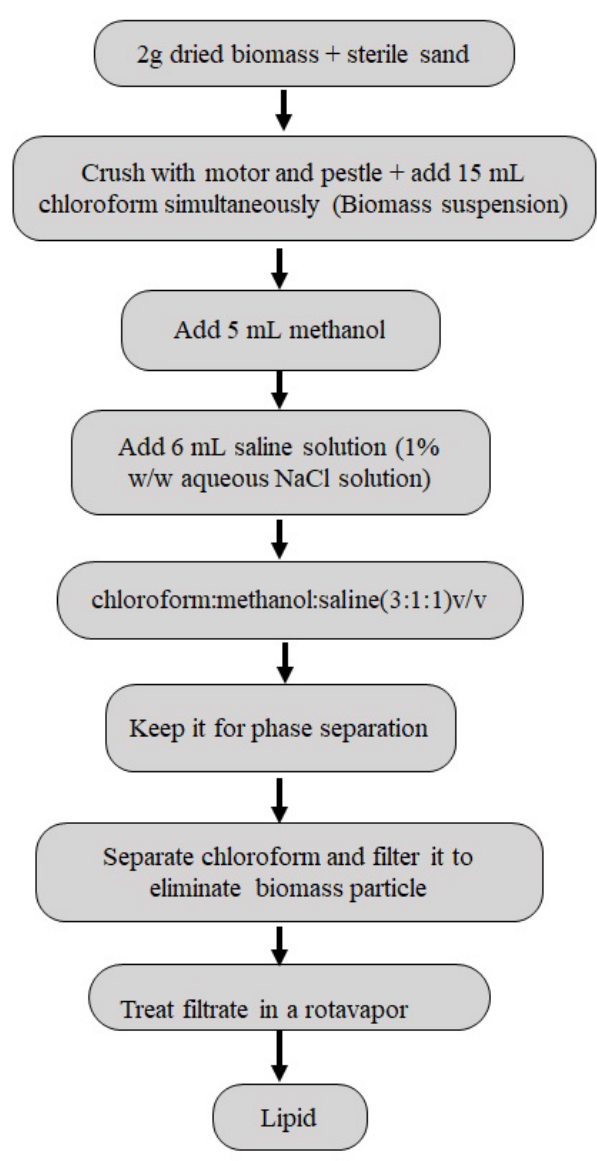

Figure 2. Diagrammatic representation of two different methods for lipid extraction. (A) Filch method and (B) Bligh and Dyer method (modified from $[29,31]$ ) 
membrane [24]. The finer resolution efficiency of immunogold labeling through electron microscopy makes it a better technology for lipid localization. Lipid-associated proteins can be identified using the following steps: (i) cell disruption, (ii) lipid body extraction, (iii) protein extraction from lipid, and (iv) protein identification [25]. In the proteomic study of $F$. solaris, cells were disrupted by multi-bead shocker [19]. The direct contact of lipids with cellular membrane creates obstacle in pure and intact protein extraction [25]. Identification of proteins can be carried out using sodium dodecyl sulfate-polyacrylamide gel electrophoresis SDSPAGE and liquid chromatography coupled with mass spectrometry [26].

\subsection{Lipid Extraction}

The steps for biodiesel production from microalgae are biomass production, lipid extraction, and transesterification of extracted lipids. Lipid extraction is an expensive process, and it can hinder biodiesel production [27]. All the steps are imperative, but cell disruptions are the most essential step as it determines the content of extracted lipid. It means the key component of lipid extraction efficiency is cell disruption method and device. Methods, such as microwaves, and bead-beating, sonication are used for cell disruption. Sonication breaks the cell wall and membrane because of cavitation effect; bead-beating causes mechanical damage to the cells [28]. Two methods are usually used to extract lipids: organic solvent and solvent-free approaches. Organic solvents have safety and environment concerns, as open emissions can dominate the potential environmental impacts of a process. However, lipid extraction from algal cells using solvent-based approaches is fast which is required for lipid extraction to avoid damage to extracted lipids. Moreover, extraction efficiency is dependent on the solvent used because polar compounds are more soluble in polar solvents. Therefore, use of the co-solvent, for instance chloroform, methanol, and n-hexane can improve the extraction efficiency and accelerate the procedure [29].

Various techniques are available for lipid extraction such as Filch method, Soxhlet extraction method, the Bligh and dyer method, a microwave oven technique, supercritical fluid extraction, ultrasound-assisted extraction, and pressurized fluid extraction. The choice of lipid extraction technique depends on several factors' species, the initial lipid content, and the amount of biomass treated per unit time [30]. In Folch method, a mixture of chloroform: methanol (2:1) is used in this method to isolate lipids from endogenous cells. In brief, the homogenized cells are mixed with saline solution and the mixture is allowed for phase separation, and it results in two layers. The upper phase contains lipids. It is one of the oldest initiatives of extraction of lipids [29]. In Bligh and dyer approach, lipids are extracted from the biomass suspension using 1:2 (v/v) chloroform/methanol. Lipids are extracted from the chloroform phase and then process by various methods (Fig. 2) [31].

\section{CONCLUSION}

This century is in dire need of alternative (e.g., biofuel) to fossil fuel due to their declining amount and negative impact on environment. Diatoms are known as promising source of biofuel because of their ability to provide great biomass yield, high rate of carbon dioxide fixation, and no competition with food crops for resources. They also generate high value co-products that are good for food and health's markets. Diatoms came into existence over last 200 million year ago. They accumulate neutral lipids in the intercellular compartment, known as lipid droplets. These neutral lipids, such as TAG, are converted into biofuels by transesterification reaction. Significant challenges are faced during processing of microalgae biomass for biodiesel production. Diatoms are a good source of bioactive compounds and can produce a large variety of lipids. The average lipid content of diatoms can reach up to $25 \%$ of their dry weight, but it can increase under stress conditions such as nutrient limitation. For instance, changes in carbon dioxide supply can improve both lipids and carbohydrates accumulation. They can be manipulated by genetic engineering to increase lipid content. Lipid droplets have association with other organelles such as mitochondria, nucleus, endosomes, endoplasmic reticulum, peroxisome, vacuoles, and plastids via direct contact, close proximity, and vesicular transport. Diatoms have high potential in biotechnology industry for lipid production. However, a complete understanding of lipid physiology, regulation, and proteomics in diatoms is required for further optimization.

\section{AUTHORS' CONTRIBUTIONS}

Rishanpreet Kaur: drafted the manuscript and concept and design; Lovepreet Kaur: drafted the manuscript; Shristy Gautam: revision of manuscript and approval.

\section{CONFLICT OF INTEREST}

The authors have declared that no conflicts of interest exist.

\section{FUNDING}

This research did not receive any specific funding.

\section{ETHICAL APPROVALS}

Not applicable.

\section{REFERENCES}

1. Battarbee RW, Jones VJ, Flower RJ, Cameron NG, Bennion H, Carvalho L, et al. Diatoms. In: Smol J.P., Birks H.J.B., Last W.M., Bradley R.S., Alverson K. (eds). Tracking environmental change using lake sediments. Springer, Berlin, Germany, pp 155-202, 2002.

2. Zulu NN, Zienkiewicz K, Vollheyde K, Feussner I. Current trends to comprehend lipid metabolism in diatoms. Prog Lipid Res 2018;70:116.

3. Li HY, Lu Y, Zheng JW, Yang WD, Liu JS. Biochemical and genetic engineering of diatoms for polyunsaturated fatty acid biosynthesis. Mar Drugs 2014;12(1):153-66.

4. Stonik V, Stonik I. Low-molecular-weight metabolites from diatoms: structures, biological roles and biosynthesis. Mar Drugs 2015;13(6):3672-709.

5. Jensen EL, Yangüez K, Carrière F, Gontero B. Storage compound accumulation in diatoms as response to elevated $\mathrm{CO} 2$ concentration. Biology 2020;9(1):5.

6. Schoefs B, Hu H, Kroth PG. The peculiar carbon metabolism in diatoms. R Soc Open Sci 2017;372:1728.

7. Leyland B, Zarka A, Didi-Cohen S, Boussiba S, Khozin-Goldberg I. High resolution proteome of lipid droplets isolated from the pennate 
diatom Phaeodactylum tricornutum (Bacillariophyceae) strain pt4 provides mechanistic insights into complex intracellular coordination during nitrogen deprivation. J Phycol 2020;56(6):1642-1663.

8. Guerra LT, Levitan O, Frada MJ, Sun JS, Falkowski PG, et al. Regulatory branch points affecting protein and lipid biosynthesis in the diatom Phaeodactylum tricornutum. Biomass Bioenergy 2013;59:306-15.

9. Rousch JM, Bingham SE, Sommerfeld MR. Changes in fatty acid profiles of thermo-intolerant and thermo-tolerant marine diatoms during temperature stress. J Exp Mar Biol Ecol 2003;295(2):145-56.

10. Yi Z, Xu M, Di X, Brynjolfsson S, Fu W. Exploring valuable lipids in diatoms. Front Mar Sci 2017;4:17.

11. Ackman RG, Jangaard P, Hoyle R, Brockerhoff H. Origin of marine fatty acids. I. Analyses of the fatty acids produced by the diatom Skeletonema costatum. Can J Fish Aquas 1964;21(4):747-56.

12. Das P, Mandal SC, Bhagabati S, Akhtar M, Singh S. Important live food organisms and their role in aquaculture. Front Aquac 2012;5(4):69-86.

13. Eizadora TY, Zendejas FJ, Lane PD, Gaucher S, Simmons BA, Lane TW. Triacylglycerol accumulation and profiling in the model diatoms Thalassiosira pseudonana and Phaeodactylum tricornutum (Baccilariophyceae) during starvation. J Appl Phycol 2009;21(6): 669-81.

14. Sanjay K, Nagendra PM, Anupama S, Yashaswi B, Deepak B. Isolation of diatom navicula cryptocephala and characterization of oil extracted for biodiesel production. Afr J Environ Sci Tech 2013;7(1):41-8.

15. Obata T, Fernie AR, Nunes-Nesi A. The central carbon and energy metabolism of marine diatoms. Metabolites 2013;3(2):325-46.

16. Yang ZK, Niu YF, Ma YH, Xue J, Zhang MH, Yang WD, et al. Molecular and cellular mechanisms of neutral lipid accumulation in diatom following nitrogen deprivation. Biotechnol Biofuels 2013;6(1):1-14.

17. Leyland B, Boussiba S, Khozin-Goldberg I. A review of diatom lipid droplets. Biology 2020;9(2):38.

18. Nonoyama T, Nojima D, Maeda Y, Noda M, Yoshino T, Matsumoto $\mathrm{M}$, et al. Proteomics analysis of lipid droplets indicates involvement of membrane trafficking proteins in lipid droplet breakdown in the oleaginous diatom Fistulifera solaris. Algal Res 2019;44:101660.

19. Nojima D, Yoshino T, Maeda Y, Tanaka M, Nemoto M, Tanaka T. Proteomics analysis of oil body-associated proteins in the oleaginous diatom. J Proteome Res 2013;12(11):5293-301.

20. Lupette J, Jaussaud A, Seddiki K, Morabito C, Brugière S, Schaller H, et al. The architecture of lipid droplets in the diatom Phaeodactylum tricornutum. Algal Res 2019;38:101415.

21. Shemesh Z, Leu S, Khozin-Goldberg I, Didi-Cohen S, Zarka A, Boussiba S. Inducible expression of Haematococcus oil globule protein in the diatom Phaeodactylum tricornutum: association with lipid droplets and enhancement of TAG accumulation under nitrogen starvation. Algal Res 2016;18:321-31.

22. Wang X, Hao TB, Balamurugan S, Yang WD, Liu JS, Dong HP, et al. A lipid droplet-associated protein involved in lipid droplet biogenesis and triacylglycerol accumulation in the oleaginous microalga Phaeodactylum tricornutum. Algal Res 2017;26:215-24.

23. Yoneda K, Yoshida M, Suzuki I, Watanabe MM. Identification of a major lipid droplet protein in a marine diatom Phaeodactylum tricornutum. Plant Cell Physiol 2016;57(2):397-406.

24. Soni KG, Mardones GA, Sougrat R, Smirnova E, Jackson CL, Bonifacino JS. Coatomer-dependent protein delivery to lipid droplets. J Cell Sci 2009;122(11):1834-41.

25. Maeda Y, Nojima D, Yoshino T, Tanaka T. Structure and properties of oil bodies in diatoms. Philos Trans R Soc B Biol Sci 2017;372(1728):20160408.

26. Huynh ML, Russell P, Walsh B. Tryptic digestion of in-gel proteins for mass spectrometry analysis. In: Tyther R., Sheehan D. (eds). Twodimensional electrophoresis protocols. Springer, Berlin, Germany, pp 507-13, 2009.

27. Cho SC, Choi WY, Oh SH, Lee CG, Seo YC, Kim JS, et al. Enhancement of lipid extraction from marine microalga, Scenedesmus associated with high-pressure homogenization process. J Biomed Biotechnol 2012;2012:359432.

28. Lee JY, Yoo C, Jun SY, Ahn CY, Oh HM. Comparison of several methods for effective lipid extraction from microalgae. Bioresour Technol 2010;101(1):S75-7.

29. Gorgich M, Mata T, Martins A, Branco-Vieira M, Caetano N Comparison of different lipid extraction procedures applied to three microalgal species. Energy Rep 2020;6:477-82.

30. Ranjan A, Patil C, Moholkar VS. Mechanistic assessment of microalgal lipid extraction. Ind Eng Chem Res 2010;49(6):2979-85.

31. Ranjith Kumar R, Hanumantha Rao P, Arumugam M. Lipid extraction methods from microalgae: a comprehensive review. Front Energy Res 2015;2:61.

32. Misra N, Panda PK, Parida BK, Mishra BK. Phylogenomic study of lipid genes involved in microalgal biofuel production - Candidate gene mining and metabolic pathway analyses. Evol Bioinform Online 2012;8:545-64.

How to cite this article:

Kaur R, Kaur L, Gautam S. Role of lipid droplets in diatoms for biofuel production. J Appl Biol Biotech 2021; 9(05): 186-191. 